This is a self-archived version of an original article. This version may differ from the original in pagination and typographic details.

Author(s): Massel, Francesco

Title: Backaction-evading measurement of entanglement in optomechanics

Year: 2019

Version: Published version

Copyright: @ 2019 American Physical Society

Rights: In Copyright

Rights url: http://rightsstatements.org/page/lnC/1.0/?language=en

Please cite the original version:

Massel, F. (2019). Backaction-evading measurement of entanglement in optomechanics.

Physical Review A, 100(2), Article 023824. https://doi.org/10.1103/PhysRevA.100.023824 


\title{
Backaction-evading measurement of entanglement in optomechanics
}

\author{
Francesco Massel (1)* \\ Department of Physics and Nanoscience Center, University of Jyväskylä, P.O. Box 35 (YFL), Jyväskylä FI-40014, Finland
}

(Received 15 February 2019; published 15 August 2019)

\begin{abstract}
We propose here a fully backaction-evading scheme for the measurement of the entanglement between two nanomechanical resonators. The system, which consists of two mechanical oscillators, coupled to a single mode of an electromagnetic resonant cavity through a radiation-pressure interaction term, is driven by two pump tones and four detection tones. As previously discussed in the literature, the former induce entanglement between the two mechanical oscillators, while we show here that a specific choice of phase and amplitude of the detection tones allows for direct pairwise reconstruction of the collective quadrature fluctuations of the mechanical oscillators belonging to quantum-mechanics-free subspaces, thereby providing direct evidence of the entanglement properties of the two mechanical resonators.
\end{abstract}

DOI: 10.1103/PhysRevA.100.023824

\section{INTRODUCTION}

The study of the properties of mechanical systems at the nanoscale represents an extremely active field of research, both at the fundamental and the applied level [1-5]. While the quantum harmonic oscillator is arguably one of the first examples encountered in the study of quantum systems, the experimental realization of a quantum state for a typical "mass + spring" mechanical system has been achieved only recently [6-9]. In the preparation and detection of quantum states for mechanical devices, the physics of cavity optomechanical systems has played a prominent role [10]. These systems allow for the manipulation of mechanical degrees of freedom through a radiation-pressure coupling acting between a mechanical resonator and an electromagnetic field within a resonant cavity. Experiments in this sense have led to the preparation of different quantum mechanical states such as the quantum ground state of a nanodrum coupled to a microwave cavity [7], and quantum-squeezed [11-13] and entangled states for nanomechanical resonators [8,9].

One of the crucial aspects concerning the manipulation of quantum mechanical degrees of freedom-in particular for optomechanical systems - is represented by the strategies aimed at certifying, through measurement, that the desired mechanical state has indeed been prepared. Within the theoretical framework of quantum measurement [14] and, more in general, of the physics of open quantum systems [15], different strategies have been proposed to control and probe the state of the optomechanical systems [7,16-22]. Of particular relevance to our analysis are the so-called backaction evading (BAE) measurement setups [23,24], which aim at circumventing the effect of the disturbance induced by the measurement apparatus on the system (backaction), potentially compromising the preparation of a given quantum state (see, e.g., Refs. $[9,25]$ for recent examples in this sense).

\footnotetext{
*francesco.p.massel@jyu.fi
}

In this paper, we propose a four-tone BAE measurement setup aimed at the characterization of the entanglement properties of two mechanical resonators. In particular, the experimental setting we discuss here is constituted by two mechanical resonators and an electromechanical cavity, either in the optical or in the microwave regime.

Among the possible measures allowing the quantification of entanglement, the most suitable for our setting is represented by the violation of the Duan bound [26]. According to this criterion, to quantify whether such system is entangled, it is necessary to establish whether the collective quadratures $X_{\Sigma}=X_{1}+X_{2}, Y_{\Delta}=Y_{1}-Y_{2}$ of the mechanical modes violate an inequality of the form

$$
\left\langle\Delta X_{\Sigma}^{2}\right\rangle+\left\langle\Delta Y_{\Delta}^{2}\right\rangle \geqslant 1,
$$

where $\left\langle\Delta X_{\Sigma}^{2}\right\rangle=\int \frac{d \omega}{2 \pi}\left\langle\left\{X_{\Sigma, \omega}, X_{\Sigma,-\omega}\right\}\right\rangle / 2$ and analogously for $\left\langle\Delta Y_{\Delta}^{2}\right\rangle$. The operators $X_{1,2}$ and $Y_{1,2}$ represent the quadrature operators for each of the two mechanical resonators and fulfill the canonical commutation relations $\left[X_{\mathrm{n}}, Y_{\mathrm{m}}\right]=i \delta_{\mathrm{n}, \mathrm{m}}(\mathrm{n}, \mathrm{m}=$ $1,2)$. Quadrature operators are proportional to the position $Q=\sqrt{\hbar /\left(m \omega_{0}\right)} X$ and momentum $P=\sqrt{\hbar m \omega_{0}} Y$ operators associated with the dynamics of a mechanical oscillator of mass $m$ and resonant frequency $\omega_{0}$. The goal of our paper is to suggest a measurement setup allowing for the BAE detection of $\left\langle\Delta X_{\Sigma}^{2}\right\rangle$ and $\left\langle\Delta Y_{\Delta}^{2}\right\rangle$.

The setting discussed here represents, on the one hand, an improvement over the detection setup utilized in the experimental verification of the entanglement between mechanical modes introduced in Ref. [9], in which $\left\langle\Delta X_{\Sigma}^{2}\right\rangle$ could be measured directly, through a BAE measurement, whereas $\left\langle\Delta Y_{\Delta}^{2}\right\rangle$ was inferred from the response of the system in the absence of detection probes. On the other hand, the measurement setup introduced here is an extension of the proposal of Ref. [27], in which the four-probe setup, while directly measuring $\left\langle\Delta X_{\Sigma}^{2}\right\rangle$ and $\left\langle\Delta Y_{\Delta}^{2}\right\rangle$, did not fulfill the BAE condition, therefore introducing extra backaction noise in the dynamics of the mechanical resonators, potentially compromising the mechanical entanglement between the oscillators. 
The paper is organized as follows: after introducing the equations of motion for the system, we propose a hierarchical solution strategy analogous to the one introduced in [27] for the fluctuation operators. Subsequently, we show how a specific choice for the probing tones provides a BAE framework for the detection of mechanical entanglement through the direct measurement of the output cavity noise spectrum.

In particular, we will show how the current choice of detection tones allows for the simultaneous BAE measurement of pairs of collective quadratures belonging to "quantummechanics-free" (qm-free) subspaces [28-30] (i.e., $X_{\Sigma}, Y_{\Delta}$ or $\left.Y_{\Sigma}, X_{\Delta}\right)$. In other terms, we will show that a BAE measurement of either $X_{\Sigma}$ or $Y_{\Delta}\left(Y_{\Sigma}\right.$ or $\left.X_{\Delta}\right)$ will not add any noise to either quadratures $X_{\Sigma}$ or $Y_{\Delta}\left(Y_{\Sigma}\right.$ or $\left.X_{\Delta}\right)$, allowing therefore for a fully BAE detection of the Duan bound.

\section{THE SYSTEM}

The system we are considering consists of a resonant electromagnetic cavity coupled to two mechanical resonators through a radiation-pressure term. In the presence of an external coherent field $E(t)$-denoting with $a, b_{1}$, and $b_{2}$ the lowering operators associated with the cavity and the mechanical modes, respectively - the Hamiltonian for the system can be written as ( $\hbar=1$ throughout)

$$
\begin{aligned}
H=\omega_{\mathrm{a}} a^{\dagger} a+ & \sum_{\mathrm{i}=1,2} \omega_{\mathrm{i}} b_{\mathrm{i}}^{\dagger} b_{\mathrm{i}}+g\left(b_{\mathrm{i}}+b_{\mathrm{i}}^{\dagger}\right) a^{\dagger} a \\
& +i\left[E(t) a^{\dagger}-E^{*}(t) a\right],
\end{aligned}
$$

where $\omega_{1}, \omega_{2}$, and $\omega_{\mathrm{a}}$ are the resonant frequencies of the two mechanical oscillators and the cavity, respectively, and $g$ is the single-photon radiation pressure coupling strength. Furthermore, we assume that the external field is constituted by a (strong) driving field and a detection tone $E(t)=E_{\text {drive }}(t)+$ $E_{\text {detect }}(t)$ where

$$
\begin{aligned}
E_{\mathrm{drive}}(t)= & \alpha_{+} e^{-i \omega_{+} t}+\alpha_{-} e^{-i \omega_{-} t}, \\
E_{\mathrm{detect}}(t)= & \left(\alpha_{\mathrm{p}+} e^{i \delta t}+\alpha_{\mathrm{q}+} e^{-i \delta t}\right) e^{-i \omega_{+} t} \\
& +\left(\alpha_{\mathrm{p}-} e^{i \delta t}+\alpha_{\mathrm{q}-} e^{-i \delta t}\right) e^{-i \omega_{-} t} .
\end{aligned}
$$

As depicted in Fig. 1(b), the external field is thus composed of six tones. Anticipating the results that we will derive below, two of them $\left(\alpha_{+}\right.$and $\alpha_{-}$at frequencies $\omega_{+}$ and $\omega_{-}$, respectively) drive the mechanical resonators into an entangled state, while the amplitude and phase of the other four $\left(\alpha_{\mathrm{p}+}, \alpha_{\mathrm{q}+}, \alpha_{\mathrm{p}-}, \alpha_{\mathrm{q}-}\right)$ are chosen in such a way as to guarantee the BAE measurement of the collective quadratures ( $X_{\Sigma}, Y_{\Sigma}, X_{\Delta}, Y_{\Delta}$ for symmetric and antisymmetric modes) of the mechanical resonators. The choice of which quadrature is being measured, and therefore which quantum-mechanicsfree subspace is being accessed (either $X_{\Sigma}, Y_{\Delta}$ or $Y_{\Sigma}, X_{\Delta}$ ), depends on the choice of the relative phase between the detection tones $\left(\alpha_{\mathrm{p}+}, \alpha_{\mathrm{q}+}, \alpha_{\mathrm{p}-}, \alpha_{\mathrm{q}-}\right)$, modified here.

In order to account for the effect of the environmental noise on the system, we introduce a description in terms of quantum Langevin equations (QLEs) [31]. Denoting with $\kappa_{\mathrm{E}}, \kappa_{\mathrm{I}}, \gamma_{1}$, and $\gamma_{2}$ the dissipation rates for internal and external losses of the (a)

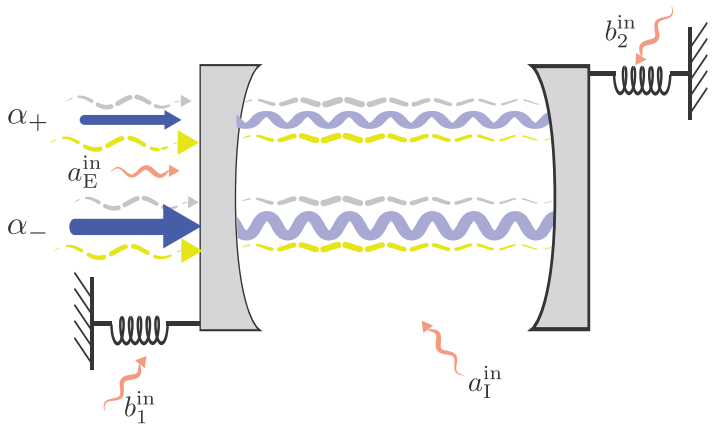

(b)

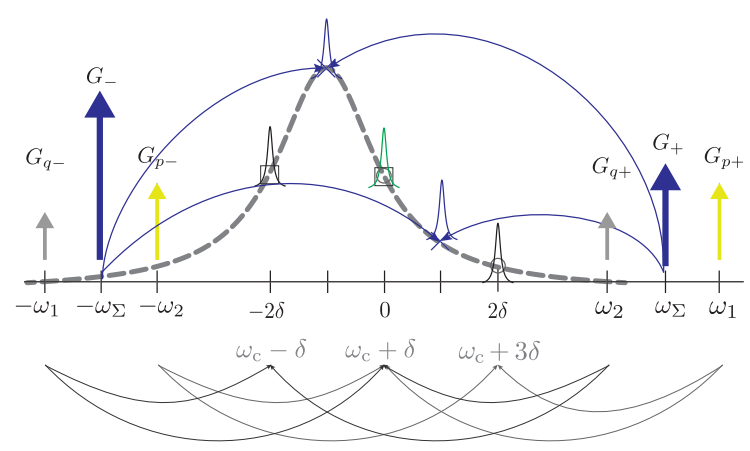

FIG. 1. (a) Sketch of the proposed entanglement detection scheme. The pump tones are depicted as blue (solid dark) arrows, while the detection tones are depicted as wiggly dashed lines. In addition, we have indicated the three sources of noise $\left(a_{\mathrm{I}}^{\text {in }}, a_{\mathrm{E}}^{\text {in }}, b^{\text {in }}\right)$ as short wiggly arrows. (b) Pictorial representation of the cavity spectrum corresponding to the choice $\omega_{+}=\omega_{1}, \omega_{-}=\omega_{2}, \omega_{\Sigma}=$ $\left(\omega_{1}+\omega_{2}\right) / 2, \delta=\left(\omega_{1}-\omega_{2}\right) / 2$ (frequency in the rotating frame; see text). The cavity mode is driven with two pumps (blue) and generates two sidebands at $\pm \delta$. In addition to the strong driving tone, we consider four probing tones (gray) which generate sidebands at $\pm 2 \delta$ and 0 . In our analysis we focus on the peak generated at 0 , which, as we will show, contains all the required information to ascertain the violation of the Duan bound.

cavity and the two mechanical resonators, respectively, we can write the QLEs in the interaction frame as

$$
\begin{aligned}
\dot{a}= & -(\kappa / 2-i \delta) a-i g\left[\left(b_{1}+b_{2}\right) e^{-i \omega_{\Sigma} t}+\left(b_{1}^{\dagger}+b_{2}^{\dagger}\right) e^{i \omega_{\Sigma} t}\right] a \\
& +E(t) e^{i\left(\omega_{a}+\delta\right) t}+\sqrt{\kappa_{\mathrm{E}}} a_{\mathrm{E}}^{\text {in }}+\sqrt{\kappa_{1}} a_{\mathrm{I}}^{\text {in }}, \\
\dot{b}_{1}= & -\left(\gamma_{1} / 2+i \delta\right) b_{1}-i g a^{\dagger} a e^{i \omega_{\Sigma} t}+\sqrt{\gamma_{1}} b_{1}^{\text {in }}, \\
\dot{b}_{2}= & -\left(\gamma_{2} / 2-i \delta\right) b_{2}-i g a^{\dagger} a e^{i \omega_{\Sigma} t}+\sqrt{\gamma_{2}} b_{2}^{\text {in }},
\end{aligned}
$$

where $a_{\mathrm{E}}^{\text {in }}, a_{\mathrm{I}}^{\text {in }}, b_{1}^{\text {in }}$, and $b_{2}^{\text {in }}$ are the input noise operators associated with the coupling of the cavity to the measurement apparatus ( $a_{\mathrm{E}}^{\text {in }}$, external noise), to the internal losses of the cavity $a_{\mathrm{I}}^{\text {in }}$, and to the thermal baths coupled to the two mechanical resonators $\left(b_{1}^{\text {in }}\right.$ and $\left.b_{2}^{\text {in }}\right)$ (see Fig. 1). The equations of motion (4a)-(4c) have been written in a rotating frame defined with respect to $H_{0}=\omega_{\Sigma}\left(b_{1}^{\dagger} b_{1}+b_{2}^{\dagger} b_{2}\right)+\left(\omega_{\mathrm{a}}+\delta\right) a^{\dagger} a$ with $\omega_{\Sigma}=$ $\left(\omega_{1}+\omega_{2}\right) / 2, \delta=\left(\omega_{1}-\omega_{2}\right) / 2$, assuming that $\omega_{ \pm}=\omega_{1,2}$.

The noise operators associated with the cavity external and internal losses $a_{i}^{\text {in }}(i=I, E)$ obey the relation $\left\langle a_{\mathrm{i}}^{\text {in }}(t) a_{\mathrm{i}}^{\text {in }} \dagger\left(t^{\prime}\right)\right\rangle=\left(n_{\mathrm{i}}+1\right) \delta\left(t-t^{\prime}\right)$, while $b_{\mathrm{j}}^{\text {in }}$ describes thermal noise for the mechanical resonator and is characterized by the correlation function $\left\langle b_{\mathrm{j}}^{\mathrm{in}}(t) b_{\mathrm{j}}^{\mathrm{in} \dagger}\left(t^{\prime}\right)\right\rangle=\left(n_{\mathrm{j}}+1\right) \delta\left(t-t^{\prime}\right)$ 
$(j=1,2)$, where $n_{\mathrm{I}, \mathrm{E}}$ and $n_{1,2}$ are the thermal occupation numbers for the "internal" and "external" cavity baths, and each mechanical resonator, respectively.

Following a standard approach, assuming that $\left|\alpha_{ \pm}\right| \gg$ $\left|\alpha_{\mathrm{p} \pm}\right|,\left|\alpha_{\mathrm{q} \pm}\right|$, Eqs. (4a)-(4c) can be linearized around the zeroth-order solution tones imposed by the driving fields as

$$
\begin{aligned}
a(t) & \rightarrow \alpha(t)+a(t), \\
b_{1,2}(t) & \rightarrow \beta_{1,2}(t)+b_{1,2}(t) .
\end{aligned}
$$

The corresponding QLEs for the fluctuations around $\alpha(t)$ and $\beta_{1,2}(t)$ become

$$
\begin{aligned}
\dot{a}= & -(\kappa / 2-i \delta) a+\sqrt{\kappa_{\mathrm{E}}} a_{\mathrm{E}}^{\text {in }}+\sqrt{\kappa_{\mathrm{I}}} a_{\mathrm{I}}^{\text {in }} \\
& -i g\left[\left(b_{1}+b_{2}\right) e^{-i \omega_{\Sigma} t}+\left(b_{1}^{\dagger}+b_{2}^{\dagger}\right) e^{i \omega_{\Sigma} t}\right] \alpha, \\
\dot{b}_{1}= & -\left(\gamma_{1} / 2+i \delta\right) b_{1}-i g\left(\alpha a^{\dagger}+\alpha^{*} a\right) e^{i \omega_{\Sigma} t}+\sqrt{\gamma_{1}} b_{1}^{\text {in }}, \\
\dot{b}_{2}= & -\left(\gamma_{2} / 2-i \delta\right) b_{2}-i g\left(\alpha a^{\dagger}+\alpha^{*} a\right) e^{i \omega_{\Sigma} t}+\sqrt{\gamma_{2}} b_{2}^{\text {in }},
\end{aligned}
$$

where we have assumed that $\delta \gg g \beta_{1,2}$. In this case $\alpha(t)$ can be written as

$$
\begin{aligned}
\alpha(t)= & \left(\alpha_{+}+\alpha_{\mathrm{p}+} e^{i \delta t}+\alpha_{\mathrm{q}+} e^{-i \delta t}\right) e^{-i \omega_{\Sigma} t} \\
& +\left(\alpha_{-}+\alpha_{\mathrm{p}-} e^{i \delta t}+\alpha_{\mathrm{q}-} e^{-i \delta t}\right) e^{i \omega_{\Sigma} t} .
\end{aligned}
$$

Assuming that the sideband-resolved condition $\left(\omega_{\Sigma} \gg \kappa\right)$ holds, neglecting terms oscillating at $\pm 2 \omega_{\Sigma}$, we can write Eqs. (6a)-(6c) as

$$
\begin{aligned}
\dot{a}= & -(\kappa / 2-i \delta) a+\sqrt{\kappa_{\mathrm{E}}} a_{\mathrm{E}}^{\text {in }}+\sqrt{\kappa_{\mathrm{I}}} a_{\mathrm{I}}^{\text {in }} \\
& -i\left[\left(G_{-}+G_{\mathrm{p}-} e^{i \delta t}+G_{\mathrm{q}-} e^{-i \delta t}\right)\left(b_{1}+b_{2}\right) .\right. \\
& \left.+.\left(G_{+}+G_{\mathrm{p}+} e^{i \delta t}+G_{\mathrm{q}+} e^{-i \delta t}\right)\left(b_{1}^{\dagger}+b_{2}^{\dagger}\right)\right], \\
\dot{b}_{1}= & -\left(\gamma_{1} / 2+i \delta\right) b_{1}+\sqrt{\gamma_{1}} b_{1}^{\text {in }} \\
& -i\left[\left(G_{-}^{*}+G_{\mathrm{p}-}^{*} e^{-i \delta t}+G_{\mathrm{q}-}^{*} e^{i \delta t}\right) a\right. \\
& \left.+\left(G_{+}+G_{\mathrm{p}+} e^{i \delta t}+G_{\mathrm{q}+} e^{-i \delta t}\right) a^{\dagger}\right], \\
\dot{b}_{2}= & -\left(\gamma_{2} / 2-i \delta\right) b_{2}+\sqrt{\gamma_{2}} b_{2}^{\text {in }} \\
& -i\left[\left(G_{-}^{*}+G_{\mathrm{p}-}^{*} e^{-i \delta t}+G_{\mathrm{q}-}^{*} e^{i \delta t}\right) a\right. \\
& \left.+\left(G_{+}+G_{\mathrm{p}+} e^{i \delta t}+G_{\mathrm{q}+} e^{-i \delta t}\right) a^{\dagger}\right],
\end{aligned}
$$

where $G_{ \pm}=g \alpha_{ \pm}\left(G_{\mathrm{q} \pm}=g \alpha_{\mathrm{q} \pm}, G_{\mathrm{p} \pm}=g \alpha_{\mathrm{p} \pm}\right)$ are the linearized optomechanical coupling rates associated with the drive and detection tones, respectively. Equations (8a)-(8c) encode the possibility of generating an entangled (two-mode squeezed) state for the two mechanical modes by means of the coupling rates $G_{ \pm}$[24,27]. The addition of the detection tones $G_{\mathrm{q}, \mathrm{p} \pm}$ allows for a full reconstruction of the collective mechanical quadratures [27]. Most importantly, in contrast to the analysis carried out in Ref. [27], $\alpha_{\mathrm{p} \pm}$ and $\alpha_{\mathrm{q} \pm}$ can be chosen in such a way as to enforce the BAE condition on either the $\left(X_{\Sigma}, Y_{\Delta}\right)$ or the $\left(Y_{\Sigma}, X_{\Delta}\right)$ qm-free subspace. To show this, we introduce a Bogolyubov unitary transformation for the mechanical operators

$$
\beta_{1}=u b_{1}+v b_{2}^{\dagger}, \quad \beta_{2}=u b_{2}+v b_{1}^{\dagger},
$$

where $u=G_{-} / \mathcal{G}$ and $v=G_{+} / \mathcal{G}$ with $\mathcal{G}=\sqrt{G_{-}^{2}-G_{+}^{2}}$. Without loss of generality, we can assume equal mechanical damping rates $\left(\gamma_{1}=\gamma_{2}=\gamma\right)$. In this case, the linearized QLE equations (8a)-(8c) can be written in terms of the Bogolyubov modes in the Fourier domain (with the convention $a_{t} \stackrel{\text { FT }}{\longrightarrow} a_{\omega}$, $\left.a_{t}^{\dagger} \stackrel{\mathrm{FT}}{\rightarrow} a_{\omega}^{\dagger}\right)$ as

$$
\begin{aligned}
\chi_{\omega+\delta}^{\mathrm{a}^{-1}} a_{\omega}= & -i \mathcal{G}\left[\beta_{1, \omega}+\beta_{2, \omega}\right]+\sqrt{\kappa_{\mathrm{E}}} a_{\mathrm{E}, \omega}^{\mathrm{in}}+\sqrt{\kappa_{\mathrm{I}}} a_{\mathrm{I}, \omega}^{\text {in }} \\
& -i\left[\mathcal{G}_{\Delta_{1}}\left\{\beta_{1, \omega-\delta}+\beta_{2, \omega-\delta}\right\}\right. \\
& \left.+\mathcal{G}_{\Delta_{3}}\left\{\beta_{1, \omega-\delta}^{\dagger}+\beta_{2, \omega-\delta}^{\dagger}\right\}\right] \\
& -i\left[\mathcal{G}_{\Delta_{2}}\left\{\beta_{1, \omega+\delta}+\beta_{2, \omega+\delta}\right\}\right. \\
& \left.+\mathcal{G}_{\Delta_{4}}\left\{\beta_{1, \omega+\delta}^{\dagger}+\beta_{2, \omega+\delta}^{\dagger}\right\}\right] \\
\chi_{\omega-\delta}^{-1} \beta_{1, \omega}= & -i \mathcal{G} a_{\omega}+\sqrt{\gamma} \beta_{1, \omega}^{\text {in }} \\
& -i\left[\mathcal{G}_{\Delta_{1}}^{*} a_{\omega+\delta}+\mathcal{G}_{\Delta_{3}} a_{\omega-\delta}^{\dagger}\right] \\
& -i\left[\mathcal{G}_{\Delta_{2}}^{*} a_{\omega-\delta}+\mathcal{G}_{\Delta_{4}} a_{\omega-\delta}^{\dagger}\right] \\
\chi_{\omega+\delta}^{-1} \beta_{2, \omega} & -i \mathcal{G}_{\omega}+\sqrt{\gamma} \beta_{2, \omega}^{\text {in }} \\
& -i\left[\mathcal{G}_{\Delta_{1}}^{*} a_{\omega+\delta}+\mathcal{G}_{\Delta_{3}} a_{\omega-\delta}^{\dagger}\right] \\
& -i\left[\mathcal{G}_{\Delta_{2}}^{*} a_{\omega-\delta}+\mathcal{G}_{\Delta_{4}} a_{\omega+\delta}^{\dagger}\right]
\end{aligned}
$$

where $\chi_{\omega}^{\mathrm{a}}=(\kappa / 2-i \omega)^{-1}$ and $\chi_{\omega}=(\gamma / 2-i \omega)^{-1}, \mathcal{G}_{\Delta_{1,2}}=$ $\left(u G_{\mathrm{p}, \mathrm{q}-}-v G_{\mathrm{p}, \mathrm{q}+}\right)$ and $\mathcal{G}_{\Delta_{3,4}}=\left(u G_{\mathrm{p}, \mathrm{q}+}-v G_{\mathrm{p}, \mathrm{q}-}\right)$. Moreover, $\beta_{1}^{\text {in }}=u b_{1}^{\text {in }}+v b_{2}^{\text {in }}$ and $\beta_{2}^{\text {in }}=u b_{2}^{\text {in }}+v b_{1}^{\text {in }^{\dagger}}$ are two correlated thermal noise operators whose only nonzero correlation functions are

$$
\begin{gathered}
\left\langle\beta_{1, \omega}^{\text {in }} \beta_{1, \omega^{\prime}}^{\text {in } \dagger}\right\rangle=\left\langle\beta_{2, \omega}^{\text {in }} \beta_{2, \omega^{\prime}}^{\text {in } \dagger}\right\rangle=\left[(n+1) u^{2}+n v^{2}+1\right] \delta_{\omega+\omega^{\prime}}, \\
\left\langle\beta_{1, \omega}^{\text {in } \dagger} \beta_{1, \omega^{\prime}}^{\text {in }}\right\rangle=\left\langle\beta_{2, \omega}^{\text {in } \dagger} \beta_{2, \omega^{\prime}}^{\text {in }}\right\rangle=\left[(n+1) v^{2}+n u^{2}\right] \delta_{\omega+\omega^{\prime}}, \\
\left\langle\beta_{1, \omega}^{\text {in }} \beta_{2, \omega^{\prime}}^{\text {in }}\right\rangle=\left\langle\beta_{1, \omega}^{\text {in } \dagger} \beta_{2, \omega^{\prime}}^{\text {in } \dagger}\right\rangle=[(2 n+1) u v] \delta_{\omega+\omega^{\prime}}
\end{gathered}
$$

where we have assumed the same thermal population for the mechanical resonators $\left(n=n_{1}=n_{2}\right)$. We now suppose that the probe tones are given by

$$
G_{\mathrm{p}, \mathrm{q} \pm}=G_{\mathrm{p}, \mathrm{q}} \exp \left[ \pm i \phi_{\mathrm{p}, \mathrm{q}}\right]
$$

with $G_{\mathrm{p}, \mathrm{q}}$ real and positive. In this case, we have that

$$
\begin{aligned}
\mathcal{G}_{\Delta_{1}} & =\mathcal{G}_{\mathrm{p}} \exp \left[-i \phi_{1}\right], \\
\mathcal{G}_{\Delta_{3}} & =\mathcal{G}_{\mathrm{p}} \exp \left[i \phi_{1}\right], \\
\mathcal{G}_{\Delta_{2}} & =\mathcal{G}_{\mathrm{q}} \exp \left[-i \phi_{2}\right], \\
\mathcal{G}_{\Delta_{4}} & =\mathcal{G}_{\mathrm{q}} \exp \left[i \phi_{2}\right],
\end{aligned}
$$

where $\quad \mathcal{G}_{\mathrm{p}, \mathrm{q}}=\left|u e^{-i \phi_{p, q}}-v e^{i \phi_{p, q}}\right| G_{\mathrm{p}, \mathrm{q}} \quad$ and $\quad \phi_{1,2}=$ $\arctan \left[\frac{u+v}{u-v} \tan \left(\phi_{\mathrm{p}, \mathrm{q}}\right)\right]$. With these assumptions, Eqs. (10a)(10c) can be solved treating the probes as perturbations with respect to the pump tones

$$
\begin{array}{ll}
\mathcal{G}_{\Delta_{1}}=\lambda \mathcal{G}_{\mathrm{p}} \exp \left[-i \phi_{1}\right], & \mathcal{G}_{\Delta_{2}}=\lambda \mathcal{G}_{\mathrm{q}} \exp \left[-i \phi_{2}\right], \\
\mathcal{G}_{\Delta_{3}}=\lambda \mathcal{G}_{\mathrm{p}} \exp \left[i \phi_{1}\right], & \mathcal{G}_{\Delta_{4}}=\lambda \mathcal{G}_{\mathrm{q}} \exp \left[i \phi_{2}\right],
\end{array}
$$

where we have introduced the formal perturbative parameter $\lambda(\lambda=1$ in the end of the calculation). The solutions for $a$, 
$\beta_{1}$, and $\beta_{2}$ can be expressed in powers of the perturbative parameter $\lambda$ as

$$
\begin{gathered}
a_{\omega}=a_{\omega}^{(0)}+\lambda a_{\omega}^{(1)}+\lambda a_{\omega}^{(2)}+O\left(\lambda^{3}\right), \\
\beta_{1, \omega}=\beta_{1, \omega}^{(0)}+\lambda \beta_{1, \omega}^{(1)}+\lambda \beta_{1, \omega}^{(2)}+O\left(\lambda^{3}\right), \\
\beta_{2, \omega}=\beta_{2, \omega}^{(0)}+\lambda \beta_{2, \omega}^{(1)}+\lambda \beta_{2, \omega}^{(2)}+O\left(\lambda^{3}\right) .
\end{gathered}
$$

Substituting the perturbative expression given in Eqs. (17a)-(17c) into Eqs. (10a)-(10c) we get that each term in the perturbative expansion can be written as

$$
\begin{aligned}
\left(\chi_{\omega+\delta}^{\mathrm{a}}\right)^{-1} a_{\omega}^{(\mathrm{n})} & =-i \mathcal{G}\left[\beta_{1, \omega}^{(\mathrm{n})}+\beta_{2, \omega}^{(\mathrm{n})}\right]+A_{\mathrm{in}}^{(\mathrm{n})}, \\
\left(\chi_{\omega-\delta}^{\mathrm{m}}\right)^{-1} \beta_{1, \omega}^{(\mathrm{n})} & =-i \mathcal{G} a_{\omega}^{(\mathrm{n})}+B_{1, \mathrm{in}}^{(\mathrm{n})}, \\
\left(\chi_{\omega+\delta}^{\mathrm{m}}\right)^{-1} \beta_{2, \omega}^{(\mathrm{n})} & =-i \mathcal{G} a_{\omega}^{(\mathrm{n})}+B_{2, \mathrm{in}}^{(\mathrm{n})}
\end{aligned}
$$

with

$$
\begin{aligned}
A_{\mathrm{in}}^{(\mathrm{n}+1)}= & -i \lambda \mathcal{G}_{\mathrm{p}}\left[e^{-i \phi_{1}}\left\{\beta_{1, \omega-\delta}^{(\mathrm{n})}+\beta_{2, \omega-\delta}^{(\mathrm{n})}\right\}+e^{i \phi_{1}}\left\{\beta_{1, \omega-\delta}^{(\mathrm{n}) \dagger}+\beta_{2, \omega-\delta}^{(\mathrm{n}) \dagger}\right\}\right] \\
& -i \lambda \mathcal{G}_{\mathrm{q}}\left[e^{-i \phi_{2}}\left\{\beta_{1, \omega+\delta}^{(\mathrm{n})}+\beta_{2, \omega+\delta}^{(\mathrm{n})}\right\}+e^{i \phi_{2}}\left\{\beta_{1, \omega+\delta}^{(\mathrm{n}) \dagger}+\beta_{2, \omega+\delta}^{(\mathrm{n}) \dagger}\right\}\right], \\
B_{1, \mathrm{in}}^{(\mathrm{n}+1)}= & -i \lambda \mathcal{G}_{\mathrm{p}}\left[a_{\omega+\delta}^{(\mathrm{n})}+a_{\omega-\delta}^{(\mathrm{n}) \dagger}\right] e^{i \phi_{1}}-i \lambda \mathcal{G}_{\mathrm{q}}\left[a_{\omega-\delta}^{(\mathrm{n})}+a_{\omega+\delta}^{(\mathrm{n}) \dagger}\right] e^{i \phi_{2}}, \\
B_{2, \mathrm{in}}^{(\mathrm{n}+1)}= & -i \lambda \mathcal{G}_{\mathrm{p}}\left[a_{\omega+\delta}^{(\mathrm{n}-1)}+a_{\omega-\delta}^{(\mathrm{n}-1) \dagger}\right] e^{i \phi_{1}}-i \lambda \mathcal{G}_{\mathrm{q}}\left[a_{\omega-\delta}^{(\mathrm{n})}+a_{\omega+\delta}^{(\mathrm{n}) \dagger}\right] e^{i \phi_{2}}
\end{aligned}
$$

for $n \geqslant 0$ and

$$
\begin{aligned}
& A_{\mathrm{in}}^{(0)}=\sqrt{\kappa_{\mathrm{E}}} a_{\mathrm{E}, \omega}^{\text {in }}+\sqrt{\kappa_{\mathrm{I}}} a_{\mathrm{I}, \omega}^{\text {in }}, \\
& B_{1, \text { in }}^{(0)}=\sqrt{\gamma} \beta_{1, \omega}^{\text {in }}, \\
& B_{2, \text { in }}^{(0)}=\sqrt{\gamma} \beta_{2, \omega}^{\text {in }} .
\end{aligned}
$$

Equations (18a)-(18c) can be solved to give

$$
\begin{aligned}
& a_{\omega}^{(\mathrm{n})}=\frac{\chi_{\omega+\delta}^{\mathrm{a}}}{\Delta}\left\{A_{\mathrm{in}}^{(\mathrm{n})}-i \mathcal{G}\left[\chi_{\omega-\delta}^{\mathrm{m}} B_{1, \mathrm{in}}^{(\mathrm{n})}+\chi_{\omega+\delta}^{\mathrm{m}} B_{2, \mathrm{in}}^{(\mathrm{n})}\right]\right\}, \\
& \beta_{1, \omega}^{(\mathrm{n})}=\frac{\chi_{\omega-\delta}^{\mathrm{m}}}{\Delta}\left\{\eta_{1} B_{1, \mathrm{in}}^{(\mathrm{n})}-i \mathcal{G}\left[\chi_{\omega}^{\mathrm{a}} A_{\mathrm{in}}^{(\mathrm{n})}-i \mathcal{G} \chi_{\omega+\delta}^{\mathrm{m}} B_{2, \mathrm{in}}^{(\mathrm{n})}\right]\right\}, \\
& \beta_{2, \omega}^{(\mathrm{n})}=\frac{\chi_{\omega+\delta}^{\mathrm{m}}}{\Delta}\left\{\eta_{2} B_{2, \mathrm{in}}^{(\mathrm{n})}-i \mathcal{G}\left[\chi_{\omega}^{\mathrm{a}} A_{\mathrm{in}}^{(\mathrm{n})}-i \mathcal{G} \chi_{\omega-\delta}^{\mathrm{m}} B_{1, \mathrm{in}}^{(\mathrm{n})}\right]\right\},
\end{aligned}
$$

where

$$
\begin{aligned}
\Delta & =1+\mathcal{G}^{2} \chi_{\omega+\delta}^{\mathrm{a}}\left(\chi_{\omega-\delta}^{\mathrm{m}}+\chi_{\omega+\delta}^{\mathrm{m}}\right), \\
\eta_{1,2} & =1+\mathcal{G}^{2} \chi_{\omega+\delta}^{\mathrm{a}} \chi_{\omega \mp \delta}^{\mathrm{m}} .
\end{aligned}
$$

For $n=0$ we have $\left(\mathcal{G}_{\mathrm{p}}=\mathcal{G}_{\mathrm{q}}=0\right)$.

$$
\begin{aligned}
a_{\omega}^{(0)}= & \frac{\chi_{\omega+\delta}^{\mathrm{a}}}{\Delta}\left\{\left[\sqrt{\kappa_{\mathrm{E}}} a_{\mathrm{E}, \omega}^{\mathrm{in}}+\sqrt{\kappa_{\mathrm{I}}} a_{\mathrm{I}, \omega}^{\mathrm{in}}\right]\right. \\
& \left.-i \mathcal{G}\left[\chi_{\omega-\delta}^{\mathrm{m}} \sqrt{\gamma} \beta_{2, \omega}^{\mathrm{in}}+\chi_{\omega+\delta}^{\mathrm{m}} \sqrt{\gamma} \beta_{2, \omega}^{\mathrm{in}}\right]\right\}, \\
\beta_{1, \omega}^{(0)}= & \frac{\chi_{\omega-\delta}^{\mathrm{m}}}{\Delta}\left\{\eta_{1} \sqrt{\gamma} \beta_{1, \omega}^{\mathrm{in}}-i \mathcal{G}\left[\chi_{\omega}^{\mathrm{a}}\left\{\sqrt{\kappa_{\mathrm{E}}} a_{\mathrm{E}, \omega}^{\mathrm{in}}+\sqrt{\kappa_{\mathrm{I}}} a_{\mathrm{I}, \omega}^{\mathrm{in}}\right\}\right.\right. \\
& \left.\left.-i \mathcal{G} \chi_{\omega+\delta}^{\mathrm{m}} \sqrt{\gamma} \beta_{2, \omega}^{\mathrm{in}}\right]\right\}, \\
\beta_{2, \omega}^{(0)}= & \frac{\chi_{\omega+\delta}^{\mathrm{m}}}{\Delta}\left\{\eta_{2} \sqrt{\gamma} \beta_{2, \omega}^{\mathrm{in}}-i \mathcal{G}\left[\chi_{\omega}^{\mathrm{a}}\left\{\sqrt{\kappa_{\mathrm{E}}} a_{\mathrm{E}, \omega}^{\mathrm{in}}+\sqrt{\kappa_{\mathrm{I}}} a_{\mathrm{I}, \omega}^{\mathrm{in}}\right\}\right.\right. \\
& \left.\left.-i \mathcal{G} \chi_{\omega-\delta}^{\mathrm{m}} \sqrt{\gamma} \beta_{1, \omega}^{\mathrm{in}}\right]\right\} .
\end{aligned}
$$

From these expressions it is possible to see that, if $\gamma \ll \delta$, $\beta_{1}^{(0)}$ and $\beta_{2}^{(0)}$ are peaked around $\omega \simeq \delta$ and $\omega \simeq-\delta$, respectively, while $a^{(0)}$ exhibits a double peak structure for $\omega \simeq \pm \delta$. Furthermore, as expected, the solution of Eqs. (23a)-(23c) allows us to establish that the original mechanical modes $b_{1}$ and $b_{2}$ are entangled, since the cooling of modes $\beta_{1}$ and $\beta_{2}$ corresponds to two-mode squeezing for $b_{1}$ and $b_{2}$.
Furthermore, we can write the $n=1$ contributions around $\omega \simeq 0$ (in the rotating frame) as

$$
\begin{aligned}
a_{\omega}^{(1)}= & -i \frac{\chi_{\omega+\delta}^{\mathrm{a}}}{\Delta}\left[\mathcal{G}_{\mathrm{p}}\left\{e^{-i \phi_{1}} \beta_{2, \omega-\delta}^{(0)}+e^{i \phi_{1}} \beta_{1, \omega-\delta}^{(0) \dagger}\right\}\right. \\
& \left.+\mathcal{G}_{\mathrm{q}}\left\{e^{-i \phi_{2}} \beta_{2, \omega+\delta}^{(0)}+e^{i \phi_{2}} \beta_{2, \omega+\delta}^{(0) \dagger}\right\}\right], \\
\beta_{1, \omega}^{(1)}= & 0, \\
\beta_{2, \omega}^{(1)}= & 0 .
\end{aligned}
$$

Equation (24a) demonstrates how the dynamics of the mechanical modes $\beta_{1}^{(0)}$ and $\beta_{2}^{(0)}$ can be inferred from the dynamics of the first-order approximation to the cavity field, and that the measurement is realized through the presence of the detection tones.

Even though the Bogolyubov operators $\beta_{1, \omega}$ and $\beta_{2, \omega}$ encode all relevant information about the dynamics of the mechanical resonators, since we are interested in the potential violation of the Duan bound (1), it is more informative to express Eqs. (24a)-(24c) in terms of frequency-shifted quadrature operators for the collective mechanical degrees of freedom $\bar{X}_{\omega}^{\Sigma}, \bar{X}_{\omega}^{\Delta}, \bar{Y}_{\omega}^{\Sigma}$, and $\bar{Y}_{\omega}^{\Delta}$, defined as

$$
\begin{aligned}
& \bar{X}_{\omega}^{\Sigma}=\bar{X}_{1, \omega}+\bar{X}_{2, \omega}, \\
& \bar{X}_{\omega}^{\Delta}=\bar{X}_{1, \omega}-\bar{X}_{2, \omega},
\end{aligned}
$$

with $\bar{X}_{1, \omega}=\left(b_{1, \omega+\delta}+b_{1, \omega-\delta}^{\dagger}\right) / \sqrt{2}$ and $\quad \bar{X}_{2, \omega}=\left(b_{2, \omega-\delta}+\right.$ $\left.b_{2, \omega+\delta}^{\dagger}\right) / \sqrt{2}$ where analogous definitions hold for $\bar{Y}_{\omega}^{\Sigma}$ and $\bar{Y}_{\omega}^{\Delta}$.

While for $\delta \neq 0$ original and shifted mechanical quadratures do not coincide, it is possible to show [27] that the uncertainties associated with the shifted mechanical quadratures $\left\langle\Delta \bar{X}_{\Sigma}^{2}\right\rangle$ and $\left\langle\Delta \bar{Y}_{\Sigma}^{2}\right\rangle$ which can be obtained integrating the mechanical noise spectra $S_{\omega}^{\Sigma, 0}$ and $S_{\omega}^{\Delta, \pi / 2}$ (Figs. 2 and 3) satisfy the following relation:

$$
\left\langle\Delta \bar{X}_{\Sigma}^{2}\right\rangle+\left\langle\Delta \bar{Y}_{\Delta}^{2}\right\rangle=\left\langle\Delta X_{\Sigma}^{2}\right\rangle+\left\langle\Delta Y_{\Delta}^{2}\right\rangle .
$$

We are thus allowed to express the Duan bound (1) in terms of frequency-shifted mechanical quadratures as

$$
\left\langle\Delta \bar{X}_{\Sigma}^{2}\right\rangle+\left\langle\Delta \bar{Y}_{\Delta}^{2}\right\rangle \leqslant 1 .
$$


From Eq. (24a) and the definiton of the shifted quadratures, it is possible to express the first-order correction to the cavity field as

$$
\begin{aligned}
a_{\omega}^{(1)}=-i \frac{\chi_{\omega+\delta}^{\mathrm{a}}}{\sqrt{2} \Delta} & {\left[\left\{\mathcal{A}_{\phi_{\mathrm{p}}, \phi_{\mathrm{q}}}^{+} \bar{X}_{\omega}^{\Sigma}+\mathcal{B}_{\phi_{\mathrm{p}}, \phi_{\mathrm{q}}}^{+} \bar{Y}_{\omega}^{\Sigma}\right\}\right.} \\
& \left.+i\left\{\mathcal{B}_{\phi_{\mathrm{p}}, \phi_{\mathrm{q}}}^{-} \bar{X}_{\omega}^{\Delta}-\mathcal{A}_{\phi_{\mathrm{p}}, \phi_{\mathrm{q}}}^{-} \bar{Y}_{\omega}^{\Delta}\right\}\right],
\end{aligned}
$$

where

$$
\begin{aligned}
& \mathcal{A}_{\phi_{\mathrm{p}}, \phi_{\mathrm{q}}}^{ \pm}=G_{\mathrm{p}} \cos \left(\phi_{\mathrm{p}}\right) \pm G_{\mathrm{q}} \cos \left(\phi_{\mathrm{q}}\right), \\
& \mathcal{B}_{\phi_{\mathrm{p}}, \phi_{\mathrm{q}}}^{ \pm}=G_{\mathrm{p}} \sin \left(\phi_{\mathrm{p}}\right) \pm G_{\mathrm{q}} \sin \left(\phi_{\mathrm{q}}\right) .
\end{aligned}
$$

To ascertain the BAE nature of the current measurement setup in each qm-free subspace, we need to evaluate the higher-order terms contributing to the cavity field around the relevant frequency ( $\omega \simeq 0$ in the rotating frame). To this end, from Eqs. (19a)-(21c), we can write

$$
\begin{aligned}
a_{\omega}^{(\mathrm{n}+2)}=-i \lambda \frac{\chi_{\omega+\delta}^{\mathrm{a}}}{\Delta} & {\left[\mathcal{G}_{\mathrm{p}}\left\{e^{-i \phi_{1}} \beta_{2, \omega-\delta}^{(\mathrm{n}+1)}+e^{i \phi_{1}} \beta_{1, \omega-\delta}^{(\mathrm{n}+1) \dagger}\right\}\right.} \\
& \left.+\mathcal{G}_{\mathrm{q}}\left\{e^{-i \phi_{2}} \beta_{1, \omega+\delta}^{(\mathrm{n}+1)}+e^{i \phi_{2}} \beta_{2, \omega+\delta}^{(\mathrm{n}+1) \dagger}\right\}\right],
\end{aligned}
$$

where, since $\gamma \ll \delta$, we have neglected all nonresonant terms in the mechanical response $\chi_{\omega}^{\mathrm{a}}$. The terms appearing on the left-hand side of Eq. (30) can, in turn, be expressed as

$$
\begin{aligned}
\beta_{1, \omega+\delta}^{(\mathrm{n}+1)}= & -i \lambda \frac{\chi_{\omega}^{\mathrm{m}}}{1+\mathcal{G}^{2} \chi_{\omega}^{\mathrm{m}} \chi_{\omega+2 \delta}^{\mathrm{a}}}\left[\mathcal{G}_{\mathrm{p}}\left(a_{\omega+2 \delta}^{(\mathrm{n})}+a_{\omega}^{(\mathrm{n}) \dagger}\right) e^{i \phi_{1}}\right. \\
& \left.+\mathcal{G}_{\mathrm{q}}\left(a_{\omega}^{(\mathrm{n})}+a_{\omega+2 \delta}^{(\mathrm{n})}\right) e^{i \phi_{2}}\right] \\
\beta_{1, \omega-\delta}^{(\mathrm{n}+1) \dagger}= & +i \lambda \frac{\chi_{\omega}^{\mathrm{m}}}{1+\mathcal{G}^{2} \chi_{\omega}^{\mathrm{m}} \chi_{\omega-2 \delta}^{\mathrm{a}}}\left[\mathcal{G}_{\mathrm{p}}\left(a_{\omega}^{(\mathrm{n})}+a_{\omega-2 \delta}^{(\mathrm{n}) \dagger}\right) e^{-i \phi_{1}}\right. \\
& \left.+\mathcal{G}_{\mathrm{q}}\left(a_{\omega-2 \delta}^{(\mathrm{n})}+a_{\omega}^{(\mathrm{n}) \dagger}\right) e^{-i \phi_{2}}\right] \\
\beta_{2, \omega-\delta}^{(\mathrm{n}+1)}= & -i \lambda \frac{\chi_{\omega}^{\mathrm{m}}}{1+\mathcal{G}^{2} \chi_{\omega}^{\mathrm{m}} \chi_{\omega}^{\mathrm{a}}}\left[\mathcal{G}_{\mathrm{p}}\left(a_{\omega}^{(\mathrm{n})}+a_{\omega+2 \delta}^{(\mathrm{n}) \dagger}\right) e^{i \phi_{1}}\right. \\
& \left.+\mathcal{G}_{\mathrm{q}}\left(a_{\omega-2 \delta}^{(\mathrm{n})}+a_{\omega}^{(\mathrm{n}) \dagger}\right) e^{i \phi_{2}}\right] \\
\beta_{2, \omega+\delta}^{(\mathrm{n}+1) \dagger}= & +i \lambda \frac{\chi_{\omega}^{\mathrm{m}}}{1+\mathcal{G}^{2} \chi_{\omega}^{\mathrm{m}} \chi_{\omega}^{\mathrm{a}}}\left[\mathcal{G}_{\mathrm{p}}\left(a_{\omega+2 \delta}^{(\mathrm{n})}+a_{\omega}^{(\mathrm{n}) \dagger}\right) e^{-i \phi_{1}}\right. \\
& \left.+\mathcal{G}_{\mathrm{q}}\left(a_{\omega}^{(\mathrm{n})}+a_{\omega+2 \delta}^{(\mathrm{n}) \dagger}\right) e^{-i \phi_{2}}\right] .
\end{aligned}
$$

Substituting Eqs. (31a)-(31d) into Eq. (30), we obtain

$$
\begin{aligned}
a_{\omega}^{(\mathrm{n}+2)}= & \lambda^{2} \frac{\chi_{\omega}^{\mathrm{m}} \chi_{\omega+\delta}^{\mathrm{a}}}{\Delta} \mathcal{G}_{\mathrm{p}} \mathcal{G}_{\mathrm{q}}\left\{e^{i\left(\phi_{1}-\phi_{2}\right)}-e^{i\left(\phi_{2}-\phi_{1}\right)}\right\} \\
& \times\left(a_{\omega-2 \delta}^{(\mathrm{n})}-a_{\omega+2 \delta}^{(\mathrm{n})}\right),
\end{aligned}
$$

implying that, for $\phi_{1}-\phi_{2}=0, \pi$, all terms $a_{\omega}^{(\mathrm{n}+2)}(n>0)$ are zero for $\delta \ll \kappa$. This condition, combined with the expression for $a_{\omega}^{(1)}$ given in Eq. (28), allows us to conclude that a choice of the detection tone phases, that fulfills the condition $\phi_{1}-\phi_{2}=$ $0, \pi$, leads to the faithful mapping onto the cavity field of the shifted quadrature field selected by the relative phase of the detection tones (Fig. 2).

\section{SPECTRUM OF THE OUTPUT FIELD}

In the previous section, we have determined that it is possible to access the information about the collective dynamics of (a) $10^{4}$

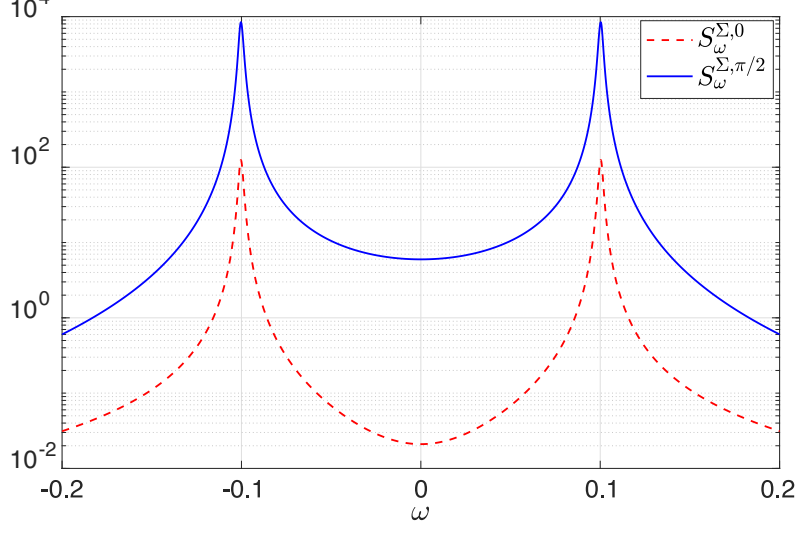

(b) $10^{4}$

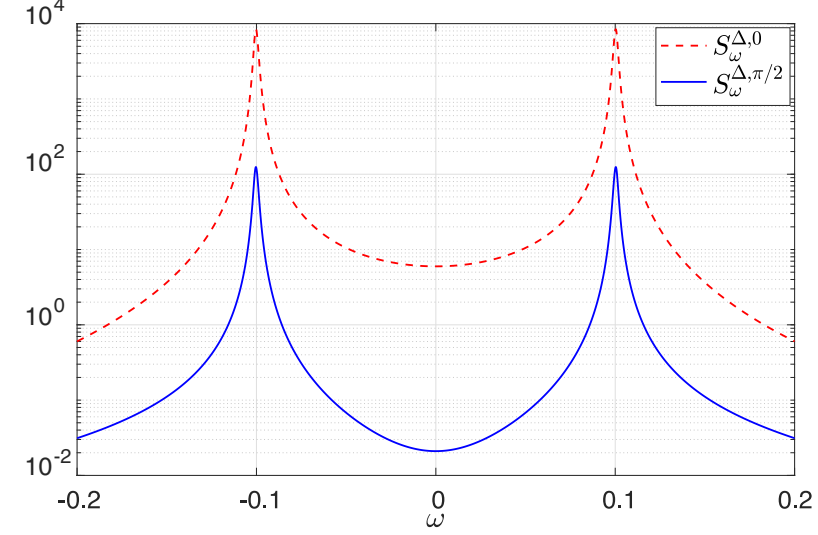

FIG. 2. (a) Noise spectrum of the symmetrical mechanical quadrature $S_{\omega}^{\Sigma, \theta}$ as a function of frequency $\omega$ for $\theta=0$ (red dotted curve) and $\theta=\pi / 2$ (blue solid curve). (b) Spectrum of the antisymmetrical mechanical quadrature $S_{\omega}^{\Delta, \theta}$ for $\theta=0$ (red dotted curve) and $\theta=\pi / 2$ (blue solid curve). Parameters are $\gamma=10^{-5}, \delta=0.1$, $G_{-}=4.8 \times 10^{-2}, G_{+}=4.0 \times 10^{-2}$, and $n_{1}=n_{2}=10, n_{\mathrm{c}}=0$. All frequencies are in units of $\kappa, \hbar=1$ throughout the paper.

the mechanical resonators through the cavity field $a_{\omega}$, which does not represent a quantity that is directly accessible in experiments. However, through the standard approach represented by the input-output formalism [31], we can relate the cavity field to the output field $a_{\omega}^{\text {out }}$-a quantity that can be measured in experiments-as $a_{\omega}^{\text {out }}=\sqrt{\kappa_{\mathrm{E}}} a_{\omega}-a_{\mathrm{E}, \omega}^{\text {in }}$. To this end, we need to evaluate the expression for the output quadrature field in terms of the perturbative expansion given in Eq. (17a). Assuming that $\phi_{1}-\phi_{2}=0$, $\pi$, we can write the output field quadratures as

$$
\begin{aligned}
X^{\text {out }, \theta}(\omega)= & {\left[\left(a_{\omega}^{(0) \text { out }}+\lambda \sqrt{k_{\mathrm{E}}} a_{\omega}^{(1)}\right) e^{-i \theta}\right.} \\
& \left.+\left(a_{\omega}^{(0) \text { out }}{ }^{\dagger}+\lambda \sqrt{k_{\mathrm{E}}} a_{\omega}^{(1)^{\dagger}}\right) e^{i \theta}\right] / \sqrt{2}
\end{aligned}
$$

and, since $\left\langle a^{(0)(\dagger)} a^{(1)(\dagger)}\right\rangle=0$, express the spectrum for the output field $S_{\omega}^{\text {out }}=\frac{1}{2}\left\langle\left\{X^{\text {out }, \theta}(\omega), X^{\text {out }, \theta}(-\omega)\right\}\right\rangle$ as

$$
S_{\omega}^{\text {out }}=S_{\omega}^{\text {out }(0)}+\kappa_{\mathrm{E}} S_{\omega}^{(1)},
$$

where

$$
\begin{aligned}
S_{\omega}^{\text {out }(0)} & =\frac{1}{2}\left\langle\left\{X^{(0) \text { out }, \theta}(\omega), X^{(0) \text { out }, \theta}(-\omega)\right\}\right\rangle \\
& =\left|\kappa_{\mathrm{E}} \chi_{\omega}^{\mathrm{a}}-1\right|^{2}\left(n_{\mathrm{c}}+\frac{1}{2}\right)
\end{aligned}
$$


(a)

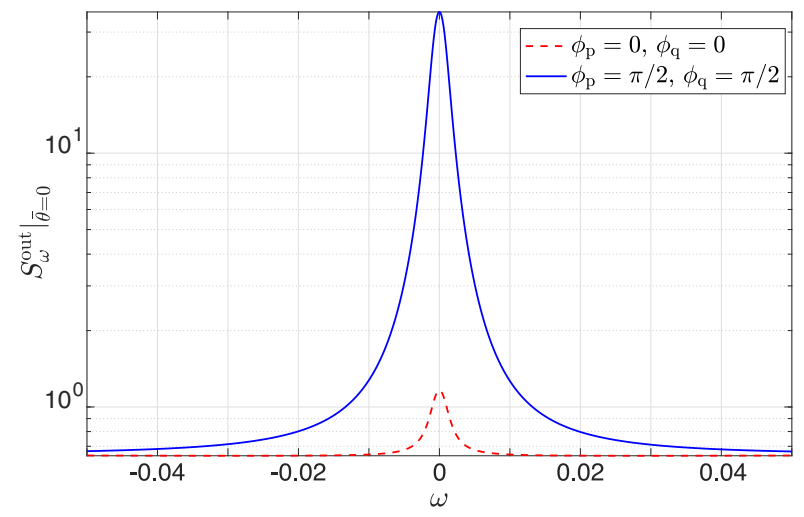

(b)

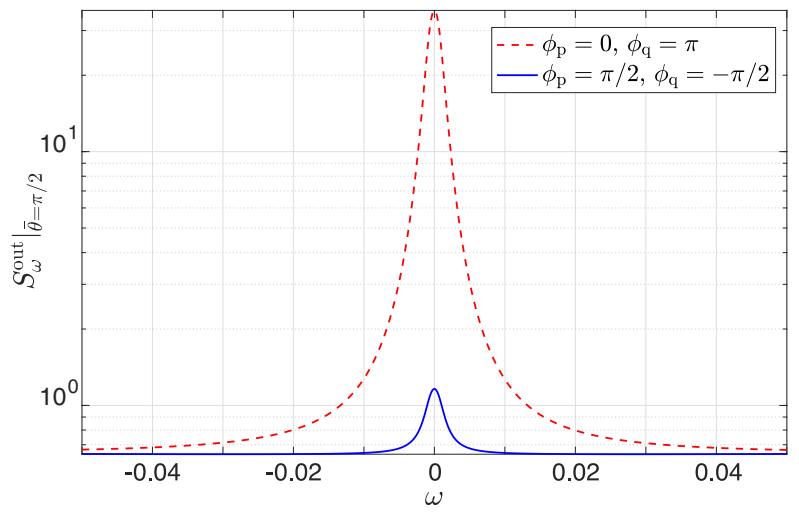

FIG. 3. (a) Output spectrum $\left.S_{\omega}^{\text {out }}\right|_{\bar{\theta}=0}$ as a function of frequency for $\phi_{\mathrm{p}}=0, \phi_{\mathrm{q}}=0$ (red dotted curve) and $\phi_{\mathrm{p}}=0, \phi_{\mathrm{q}}=0$ (blue solid curve). (b) Output spectrum $\left.S_{\omega}^{\text {out }}\right|_{\bar{\theta}=\pi / 2}$ as a function of frequency for $\phi_{\mathrm{p}}=0, \phi_{\mathrm{q}}=\pi$ (red dotted curve) and $\phi_{\mathrm{p}}=\pi / 2, \phi_{\mathrm{q}}=$ $-\pi / 2$ (blue solid curve). $\kappa_{\mathrm{E}}=0.9$; all other parameters as in Fig. 2 . The quantity appearing in the Duan inequality given by Eq. (1) can be inferred from the area under the red dotted curve in (a) and the blue solid curve in (b).

represents the contribution to the output field noise spectrum in the absence of coupling to the mechanical motion $\left(\mathrm{G}, \mathcal{G}_{\mathrm{p}, \mathrm{q}}=0\right)$ (Fig. 3).

More interestingly, $S^{(1)}(\omega)$ is the contribution to the output field noise spectrum due to the dynamics of the mechanical oscillators and therefore represents the relevant term for the determination of a potential violation of the Duan inequality. From Eq. (28), we have

$$
\begin{aligned}
S^{(1)}(\omega)= & \left|\frac{\chi_{\omega+\delta}^{\mathrm{a}}}{\Delta}\right|^{2} \\
& \times\left\{\cos ^{2}(\theta)\left[\left(\mathcal{A}_{\phi_{\mathrm{p}}, \phi_{\mathrm{q}}}^{+}\right)^{2} \bar{S}_{\omega}^{\Sigma, 0}+\left(\mathcal{B}_{\phi_{\mathrm{p}}, \phi_{\mathrm{q}}}^{+}\right)^{2} \bar{S}_{\omega}^{\Sigma, \pi / 2}\right]\right. \\
& \left.+\sin ^{2}(\theta)\left[\left(\mathcal{B}_{\phi_{\mathrm{p}}, \phi_{\mathrm{q}}}^{-}\right)^{2} \bar{S}_{\omega}^{\Delta, 0}+\left(\mathcal{A}_{\phi_{\mathrm{p}}, \phi_{\mathrm{q}}}^{-}\right)^{2} \bar{S}_{\omega}^{\Delta, \pi / 2}\right]\right\},
\end{aligned}
$$

where $\quad \bar{S}_{\omega}^{\Sigma, \Delta, 0}=\left\langle\left\{\bar{X}_{-\omega}^{\Sigma, \Delta}, \bar{X}_{\omega}^{\Sigma, \Delta}\right\}\right\rangle / 2 \quad$ and $\quad \bar{S}_{\omega}^{\Sigma, \Delta, \pi / 2}=$ $\left\langle\left\{\bar{Y}_{-\omega}^{\Sigma, \Delta}, \bar{Y}_{\omega}^{\Sigma, \Delta}\right\}\right\rangle / 2$ are the noise spectra of the frequencyshifted collective mechanical quadratures, which, upon integration, yield the quantities needed for the determination of the violation of the Duan bound (see Fig. 4). From Eq. (36), and the expressions of $\mathcal{A}_{\phi_{\mathrm{p}}, \phi_{\mathrm{q}}}^{ \pm}$and $\mathcal{B}_{\phi_{\mathrm{p}}, \phi_{\mathrm{q}}}^{ \pm}$given

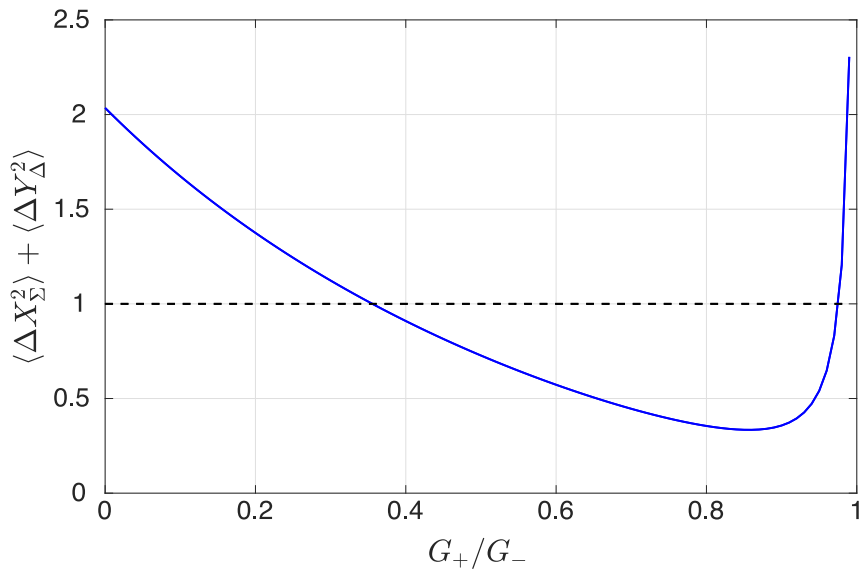

FIG. 4. Plot of the Duan quantity in Eq. (1) as a function of ratio $G_{+} / G_{-}$. The dashed line indicates the threshold below which the Duan inequality is violated. All parameters except $G_{+}$as in Figs. 2 and 3. As discussed in the text, the value of the Duan quantity can be extracted from the output spectrum as the sum of the integral under the red dotted curve in Fig. 3(a) and the blue solid curve in Fig. 3(b). See also Table I. The values of $\phi_{\mathrm{p}}$ and $\phi_{\mathrm{q}}$ chosen here imply in all cases that $\phi_{1}-\phi_{2}=0, \pi$, since $\phi_{1,2}=\arctan \left[\frac{u+v}{u-v} \tan \left(\phi_{\mathrm{p}, \mathrm{q}}\right)\right]$.

in Eqs. (29a) and (29b), it is clear that the noise spectra of the collective mechanical quadratures can be accessed from the spectrum of the output field by changing the phase of the homodyne detector $\theta$ and the phases of the detection tones $\phi_{\mathrm{p}}$ and $\phi_{\mathrm{q}}$ (compatibly with the condition $\left.\phi_{1}-\phi_{2}=0, \pi\right)$ (Fig. 4). In Table I we have summarized the different combinations of $\left(\bar{\theta}, \phi_{\mathrm{p}}, \phi_{\mathrm{q}}\right)$ allowing us to access the different frequency-shifted mechanical spectra, which, upon integration, provide a measurement of the collective mechanical quadratures needed to ascertain the violation of the Duan bound.

Furthermore, the choice of $\phi_{\mathrm{p}}, \phi_{\mathrm{q}}$, in addition to setting the mechanical quadrature to be measured, fixes the backaction induced by the measurement tones on it-expressed here as perturbative corrections- to be zero. This can be shown by

TABLE I. Relation between the output spectrum and the shifted mechanical quadrature spectra for different values of detection and probe phases.

\begin{tabular}{ccc}
\hline \hline & $\bar{\theta}=0$ & \\
1 & $\phi_{\mathrm{p}}=0$ & $S^{(1)}(\omega)=\left|\frac{\chi_{\delta}^{\mathrm{a}}}{\Delta}\right|^{2}\left(G_{\mathrm{p}}+G_{\mathrm{q}}\right)^{2} \bar{S}_{\omega}^{\Sigma, 0}$ \\
$\phi_{\mathrm{q}}=0$ & \\
& $\bar{\theta}=0$ & \\
& $\phi_{\mathrm{p}}=\pi / 2$ & $S^{(1)}(\omega)=\left|\frac{\chi_{\delta}^{\mathrm{a}}}{\Delta}\right|^{2}\left(G_{\mathrm{p}}+G_{\mathrm{q}}\right)^{2} \bar{S}_{\omega}^{\Sigma, \pi / 2}$ \\
$\phi_{\mathrm{q}}=\pi / 2$ & \\
& $\bar{\theta}=\pi / 2$ & \\
& $\phi_{\mathrm{p}}=0$ & \\
& $\phi_{\mathrm{q}}=\pi$ & \\
& $\bar{\theta}=\pi / 2$ & \\
& $\phi_{\mathrm{p}}=\pi / 2$ & $S^{(1)}(\omega)=\left|\frac{\chi_{\delta}^{\mathrm{a}}}{\Delta}\right|^{2}\left(G_{\mathrm{p}}+G_{\mathrm{q}}\right)^{2} \bar{S}_{\omega}^{\Delta, \pi / 2}\left(G_{\mathrm{p}}+G_{\mathrm{q}}\right)^{2} \bar{S}_{\omega}^{\Delta, 0}$ \\
& $\phi_{\mathrm{q}}=-\pi / 2$ & \\
\hline \hline
\end{tabular}


considering the nth-order perturbative term for the shifted quadrature operators

$$
\begin{aligned}
& \bar{X}_{\omega}^{\Sigma,(\mathrm{n})}=\frac{u-v}{\sqrt{2}}\left(\beta_{1, \omega+\delta}^{(\mathrm{n})}+\beta_{1, \omega-\delta}^{(\mathrm{n}) \dagger}+\beta_{2, \omega-\delta}^{(\mathrm{n})}+\beta_{2, \omega+\delta}^{(\mathrm{n}) \dagger}\right), \\
& \bar{X}_{\omega}^{\Delta,(\mathrm{n})}=\frac{u+v}{\sqrt{2}}\left(\beta_{1, \omega+\delta}^{(\mathrm{n})}+\beta_{1, \omega-\delta}^{(\mathrm{n}) \dagger}-\beta_{2, \omega-\delta}^{(\mathrm{n})}-\beta_{2, \omega+\delta}^{(\mathrm{n}) \dagger}\right), \\
& \bar{Y}_{\omega}^{\Sigma,(\mathrm{n})}=-i \frac{u+v}{\sqrt{2}}\left(\beta_{1, \omega+\delta}^{(\mathrm{n})}-\beta_{1, \omega-\delta}^{(\mathrm{n}) \dagger}+\beta_{2, \omega-\delta}^{(\mathrm{n})}-\beta_{2, \omega+\delta}^{(\mathrm{n}) \dagger}\right), \\
& \bar{Y}_{\omega}^{\Delta,(\mathrm{n})}=i \frac{u-v}{\sqrt{2}}\left(\beta_{1, \omega+\delta}^{(\mathrm{n})}+\beta_{1, \omega-\delta}^{(\mathrm{n}) \dagger}-\beta_{2, \omega-\delta}^{(\mathrm{n})}-\beta_{2, \omega+\delta}^{(\mathrm{n}) \dagger}\right),
\end{aligned}
$$

which, for $n>0$, represent the backaction contribution to the different quadrature operators. Setting $\phi_{\mathrm{p}}, \phi_{\mathrm{q}}$ in order to measure a given quadrature [Eq. (28)] sets the value of the backaction contributions to the mechanical quadratures [Eqs. (31a)-(31d)].

As an example one can choose $\phi_{\mathrm{p}}=\phi_{\mathrm{q}}=0$. As can be seen from Table I, this choice allows one to measure the $X_{\omega}^{\Sigma}$ quadrature. In turn, substituting the value of the mechanical Bogolyubov operators from Eqs. (31a)-(31d) with $\phi_{\mathrm{p}}=\phi_{\mathrm{q}}=0$ into Eq. (37a), one can show that $\bar{X}_{\omega}^{\Sigma,(\mathrm{n}+1)}=0$, demonstrating that the measurement is backaction evading. At the same time from Eqs. (37b)-(37d), the choice $\phi_{\mathrm{p}}=\phi_{\mathrm{q}}=0$ also entails that $\bar{Y}_{\omega}^{\Delta,(\mathrm{n}+1)}=0$, while $\bar{X}_{\omega}^{\Delta,(\mathrm{n}+1)}, \bar{Y}_{\omega}^{\Sigma,(\mathrm{n}+1)} \neq 0$.

Analogous relations hold for the different choices of $\phi_{\mathrm{p}}, \phi_{\mathrm{q}}$ giving access, depending on the value of the detection phases, to the $\left(X^{\Sigma}, Y^{\Delta}\right)$ or the $\left(X^{\Delta}, Y^{\Sigma}\right)$ qm-free subspace in a fully BAE way.

\section{CONCLUSION}

In this work, we have introduced a four-probe setup aimed at the measurement of the entanglement between two mechanical resonators in an optomechanical system, which is generated by two coherent fields driving the system into a two-mode squeezed state. We have shown that, if the probing tones are chosen correctly, within each collective qm-free subspace, no measurement backaction is present. Furthermore, selecting specific values of the probe phases, the noise spectrum of each collective mechanical quadrature can be directly mapped onto the output field noise spectrum. We would like to stress that, while we focused here on the detection of the entangled state of two mechanical resonators, the double-BAE detection scheme proposed here is actually independent of the preparation scheme of the mechanical state, therefore hinting at the possibility of a general BAE characterization of a mechanical systems dynamics within a quantum-mechanics-free subspace.

\section{ACKNOWLEDGMENT}

The author thanks M. Sillanpää and M. Asjad for useful discussions. This work was supported by the Academy of Finland (Contract No. 275245).
[1] M. Maldovan, Nature (London) 503, 209 (2013).

[2] V. Singh, S. J. Bosman, B. H. Schneider, Y. M. Blanter, A. Castellanos-Gomez, and G. A. Steele, Nat. Nanotechnol. 9, 820 (2014).

[3] R. De Alba, F. Massel, I. R. Storch, T. S. Abhilash, A. Hui, P. L. McEuen, H. G. Craighead, and J. M. Parpia, Nat. Nanotech 11, 741 (2016).

[4] P. Arrangoiz-Arriola, E. A. Wollack, M. Pechal, J. D. Witmer, J. T. Hill, and A. H. Safavi-Naeini, Phys. Rev. X 8, 031007 (2018).

[5] K. J. Satzinger, Y. P. Zhong, H.-S. Chang, G. A. Peairs, A. Bienfait, Ming-Han Chou, A. Y. Cleland, C. R. Conner, É. Dumur, J. Grebel et al., Nature (London) 563, 661 (2018).

[6] A. D. O'Connell, M. Hofheinz, M. Ansmann, R. C. Bialczak, M. Lenander, E. Lucero, M. Neeley, D. Sank, H. Wang, M. Weides, J. Wenner, J. M. Martinis, and A. N. Cleland, Nature (London) 464, 697 (2010).

[7] J. D. Teufel, T. Donner, D. Li, J. W. Harlow, M. S. Allman, K. Cicak, A. J. Sirois, J. D. Whittaker, K. W. Lehnert, and R. W. Simmonds, Nature (London) 475, 359 (2011).

[8] R. Riedinger, A. Wallucks, I. Marinković, C. Löschnauer, M. Aspelmeyer, S. Hong, and S. Gröblacher, Nature (London) 556, 473 (2018).

[9] C. F. Ockeloen-Korppi, E. Damskägg, J.-M. Pirkkalainen, M. Asjad, A. A. Clerk, F. Massel, M. J. Woolley, and M. A. Sillanpää, Nature (London) 556, 478 (2018).

[10] M. Aspelmeyer, T. J. Kippenberg, and F. Marquardt, Rev. Mod. Phys. 86, 1391 (2014).
[11] E. E. Wollman, C. U. Lei, A. J. Weinstein, J. Suh, A. Kronwald, F. Marquardt, A. A. Clerk, K. C. Schwab, Science 349, 952 (2015).

[12] J.-M. Pirkkalainen, E. Damskägg, M. Brandt, F. Massel, and M. A. Sillanpää, Phys. Rev. Lett. 115, 243601 (2015).

[13] F. Lecocq, J. B. Clark, R. W. Simmonds, J. Aumentado, and J. D. Teufel, Phys. Rev. X 5, 041037 (2015).

[14] H. M. Wiseman and G. J. Milburn, Quantum Measurement and Control (Cambridge University Press, Cambridge, 2010).

[15] C. W. Gardiner and P. Zoller, Quantum Noise (Springer, Berlin/Heidelberg/New York, 2004).

[16] S. Mancini, D. Vitali, and P. Tombesi, Phys. Rev. Lett. 80, 688 (1998).

[17] S. Mancini, V. Giovannetti, D. Vitali, and P. Tombesi, Phys. Rev. Lett. 88, 120401 (2002).

[18] D. Vitali, S. Gigan, A. Ferreira, H. R. Böhm, P. Tombesi, A. Guerreiro, V. Vedral, A. Zeilinger, and M. Aspelmeyer, Phys. Rev. Lett. 98, 030405 (2007).

[19] F. Marquardt, J. P. Chen, A. A. Clerk, and S. M. Girvin, Phys. Rev. Lett. 99, 093902 (2007).

[20] L. F. Buchmann, S. Schreppler, J. Kohler, N. Spethmann, and D. M. Stamper-Kurn, Phys. Rev. Lett. 117, 030801 (2016).

[21] M. Rossi, D. Mason, J. Chen, Y. Tsaturyan, and A. Schliesser, Nature (London) 563, 53 (2018).

[22] C. F. Ockeloen-Korppi, E. Damskägg, G. S. Paraoanu, F. Massel, and M. A. Sillanpää, Phys. Rev. Lett. 121, 243601 (2018).

[23] M. J. Woolley and A. A. Clerk, Phys. Rev. A 87, 063846 (2013).

[24] M. J. Woolley and A. A. Clerk, Phys. Rev. A 89, 063805 (2014). 
[25] C. F. Ockeloen-Korppi, E. Damskägg, J.-M. Pirkkalainen, A. A. Clerk, M. J. Woolley, and M. A. Sillanpää, Phys. Rev. Lett. 117, 140401 (2016).

[26] L.-M. Duan, G. Giedke, J. I. Cirac, and P. Zoller, Phys. Rev. Lett. 84, 2722 (2000).

[27] F. Massel, Phys. Rev. A 95, 063816 (2017).

[28] M. Tsang and C. M. Caves, Phys. Rev. X 2, 031016 (2012).
[29] E. S. Polzik and K. Hammerer, Ann. Phys. (NY) 527, A15 (2015).

[30] C. B. Møller, R. A. Thomas, G. Vasilakis, E. Zeuthen, Y. Tsaturyan, M. Balabas, K. Jensen, A. Schliesser, K. Hammerer, and E. S. Polzik, Nature (London) 547, 191 (2017).

[31] D. F. Walls and G. J. Milburn, Quantum Optics (Springer, Berlin/Heidelberg/New York, 2008). 